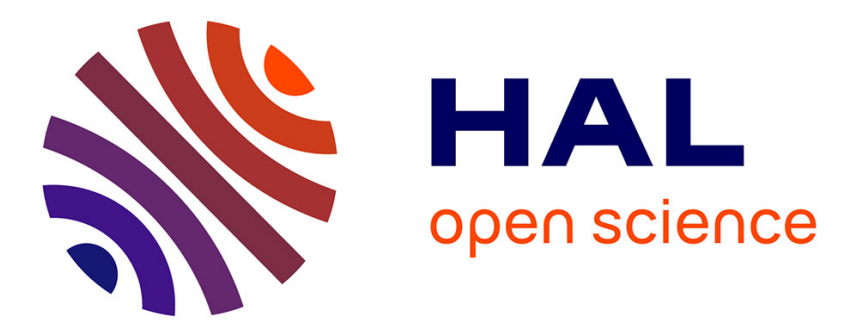

\title{
Specificity of Counterion Binding to a Conjugated Polyelectrolyte: A Combined Molecular Dynamics and NOESY Investigation
}

Gregor Hostnik, Črtomir Podlipnik, Guillaume Mériguet, Janez Cerar

\section{- To cite this version:}

Gregor Hostnik, Črtomir Podlipnik, Guillaume Mériguet, Janez Cerar. Specificity of Counterion Binding to a Conjugated Polyelectrolyte: A Combined Molecular Dynamics and NOESY Investigation. Macromolecules, 2020, 53 (4), pp.1119-1128. 10.1021/acs.macromol.9b02161 . hal-02497554

\section{HAL Id: hal-02497554 https: / hal.sorbonne-universite.fr/hal-02497554}

Submitted on 15 Dec 2021

HAL is a multi-disciplinary open access archive for the deposit and dissemination of scientific research documents, whether they are published or not. The documents may come from teaching and research institutions in France or abroad, or from public or private research centers.
L'archive ouverte pluridisciplinaire HAL, est destinée au dépôt et à la diffusion de documents scientifiques de niveau recherche, publiés ou non, émanant des établissements d'enseignement et de recherche français ou étrangers, des laboratoires publics ou privés. 


\title{
Specificity of Counterion Binding to a Conjugated Polyelectrolyte: A Combined Molecular Dynamics and NOESY Investigation
}

\author{
Gregor Hostnik, Črtomir Podlipnik, Guillaume Mériguet, and Janez Cerar* \\ Cite This: https://dx.doi.org/10.1021/acs.macromol.9b02161 \\ Read Online
}

ACCESS |

Llll Metrics \& More

Article Recommendations

Supporting Information

ABSTRACT: Poly(thiophen-3-ylacetic acid) (PTAA) is a representative of conjugated polyelectrolytes which are used in many optoelectronics devices. The performance of these devices is affected by the polymer conformation, which, among others, depends on the nature of the counterion. In this study, the binding of tetrabutylammonium counterions $\left(\mathrm{TBA}^{+}\right)$on PTAA was determined using a combination of nuclear Overhauser effect spectroscopy (NOESY) and molecular dynamics (MD) simulation. It was found that $\mathrm{TBA}^{+}$ions specifically bind on the hydrophobic main chain of PTAA, while, according to $\mathrm{MD}$
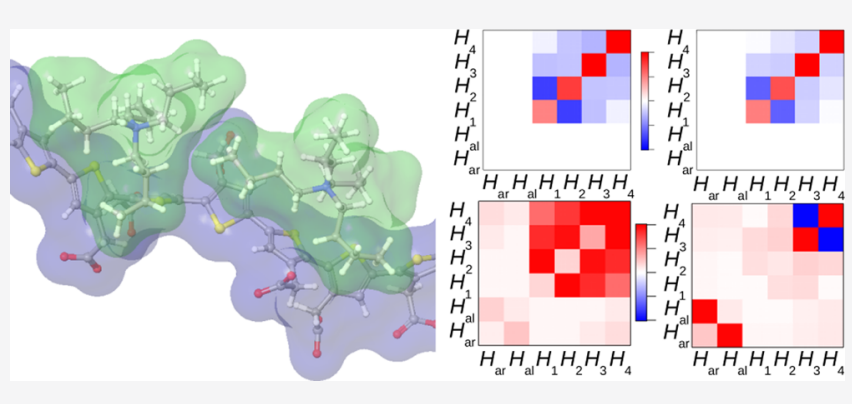
simulations, alkali counterions predominantly bind in the vicinity of negatively charged carboxylic groups located on side chains. The MD trajectories were used to compute the relaxation matrices and the NOESY spectra. With the help of these latter calculations, the changes of intensities in experimental NOESY spectra upon binding of $\mathrm{TBA}^{+}$ions to PTAA were interpreted.

\section{INTRODUCTION}

Poly(thiophen-3-ylacetic acid) (denoted further by PTAA), due to its conjugated polymer main chain and ionizable carboxylic side groups (see Figure 1), belongs to the class of conjugated
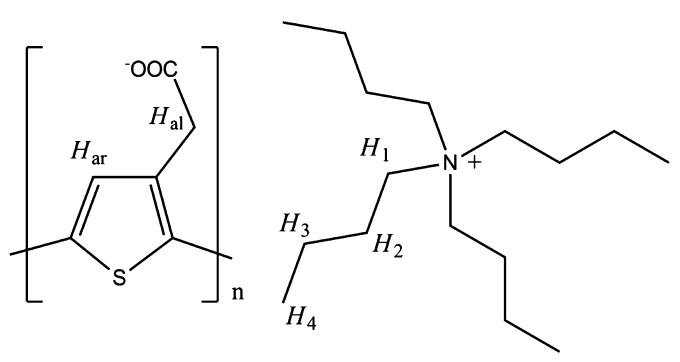

Figure 1. PTAA monomeric unit (left) neutralized with a tetrabutylammonium counterion (right). Notation of chemically distinct hydrogen atoms is added to the structure for further reference.

polyelectrolytes. Because of their interesting physical properties, such as semiconducting main chain, solubility in common polar solvents (water), and absorption of light in the ultraviolet and visible part of the spectrum, thiophene-based conjugated polymers are subjects of numerous theoretical and applied studies. $^{1-6}$

One of the crucial properties of polyelectrolytes is their conformation. ${ }^{7}$ Changes in conformation of conjugated polymers are often reflected in their spectral properties. 8,9 The conformation of a conjugated polyion is influenced by counterions present in the system. This effect can be exploited in design of different optoelectronic devices and sensors.
Understanding of interactions between the conjugated polyelectrolyte and counterions is therefore of crucial importance. In our previous studies, we investigated interactions of alkali metal and tetraalkylammonium (TAA) counterions, with PTAA using isothermal titration calorimetry (heats of mixing and dilution $)^{10,11}$ being supplemented by determination of self-diffusion coefficients using nuclear magnetic resonance (NMR) measurements. ${ }^{11}$ For alkali counterions, we have shown that the degree of binding is independent of the nature of the counterion and that the differences in heats of mixing and dilution are correlated with hydration enthalpies of these counterions. For TAA counterions, the degree of binding increases with the size of the counterion while the opposite trend is observed for the correlation between the heats of mixing and the hydration enthalpy. On the basis of these results, we proposed that the higher fraction of bound counterions and reversed dependence on the hydration enthalpy is a consequence of a different binding mechanism, very likely based on the hydrophobic interactions between PTAA and TAA ions.

In order to verify the abovementioned assumption, we designed the present study in which we tried to get deeper insight into the binding mechanism through the use of molecular dynamics (MD) simulations in combination with NMR spectroscopy. MD simulations give access to the atomic trajectories of a system for a given force field, what makes

Received: October 11, 2019

Revised: December 11, 2019 
them a perfectly suited method to determine the effect of intermolecular interactions on binding processes and the related dynamics. ${ }^{12,13}$ Experimentally, only few methods allow both the determination of the location and quantification of binding sites. Taking advantage of its chemical resolution and many varieties, NMR spectroscopy is one of such frequently used methods. ${ }^{14-17}$ In the present study, we will use nuclear Overhauser effect spectroscopy (NOESY) in combination with MD simulations. NOESY, ${ }^{18}$ and sometimes heteronuclear $\mathrm{NOESY}^{19}$ is an efficient tool to investigate the structure and conformation of molecules. It is widely used to determine the intramolecular distance under the implicit assumption that the molecules are rigid. It is used, for example, to assess the conformation of polymers. ${ }^{20}$ Furthermore, NOESY is also employed to evaluate the nature of interaction and association ${ }^{17}$ between the solvent and solute, ${ }^{21-23}$ between organic counterions and micelles, ${ }^{24}$ and between simple ions and solid polymer electrolytes. ${ }^{25}$ Regarding polyelectrolyte solutions, taking advantage of NOESY, the binding of aromatic counterions either to aromatic polyions ${ }^{26}$ or to aliphatic poly(allylammonium) cations ${ }^{27}$ was investigated. However, because the distance between the polyelectrolyte and the counterion is changing constantly, the quantitative elucidation of the NOESY spectra is achieved from the use of MD simulations. ${ }^{28-33}$

Bearing all this in mind, the binding of tetrabutylammonium counterions $\left(\mathrm{TBA}^{+}\right)$to PTAA was studied by measuring the NOESY spectrum of tetrabutylammonium salt of PTAA (PTATBA) aqueous solution and comparing the obtained spectrum with the NOESY spectrum of tetrabutylammonium chloride (TBACl) aqueous solution. Both NOESY spectra (i.e., of PTATBA and TBACl) were additionally calculated also from $\mathrm{MD}$ trajectories in order to explain the observed differences in the spectra. For comparison and for elucidation of specificity of ion binding, $\mathrm{MD}$ simulations were carried out also for aqueous solutions of alkali metal salts of PTAA.

\section{EXPERIMENTAL PART}

Synthesis and Sample Preparation. Synthesis. A detailed description of synthesis, characterization, and purification of PTAA is given in our previous papers, ${ }^{34,35}$ consequently only the most important information is repeated here. PTAA was synthesized by oxidative polymerization of thiophen-3-ylacetic acid methyl ester which was obtained by esterification of thiophen-3-ylacetic acid. After polymerization, a polymer of desired molar mass was obtained on the basis of molar mass-dependent solubility. Finally, the polymer was obtained by alkali hydrolysis with $\mathrm{NaOH}$ of the fraction with weight average molar mass $M_{\mathrm{w}}=13.4 \mathrm{~kg} \mathrm{~mol}^{-1}$ and number average molar mass $M_{\mathrm{n}}=7.2 \mathrm{~kg}$ $\mathrm{mol}^{-1}$ (dispersity $\mathrm{D}=M_{\mathrm{w}} / M_{\mathrm{n}}=1.86$ ).

Sample Preparation. For the NOESY experiment, the polymer was converted to sodium salt of PTAA by addition of $0.1 \mathrm{~mol} \mathrm{~L}^{-1} \mathrm{NaOH}$ and dialyzed against triply distilled water. The sodium salt of PTAA was converted to PTATBA by ion exchange chromatography. Afterward, water was removed by lyophilization (freeze-drying). The $0.02 \mathrm{~mol} \mathrm{~L}^{-1}$ solution of PTATBA was finally prepared by dissolution in heavy water $\left(\mathrm{D}_{2} \mathrm{O}\right)$. The concentration of obtained solution was confirmed by UV/ vis absorption spectroscopy. ${ }^{34}$ TBACl was purchased from SigmaAldrich and used as received without further purification. The $0.02 \mathrm{~mol}$ $\mathrm{L}^{-1}$ solution was prepared by dissolving a proper amount of $\mathrm{TBACl}$ in $\mathrm{D}_{2} \mathrm{O}$.

NOESY Spectra. A Bruker AVANCE DRX 500 NMR spectrometer operating at $499.76 \mathrm{MHz}$ for ${ }^{1} \mathrm{H}$ with a ${ }^{1} \mathrm{H} / \mathrm{X}$ BBO broadband probe was used for measurement of NOESY spectra. The mixing time was determined by a series of NOESY experiments with a lower resolution in the $f_{1}$ dimension on $0.02 \mathrm{~mol} \mathrm{~L}^{-1}$ PTATBA solution. The optimal mixing time, that is, the delay during which the magnetization transfer via cross-relaxation occurs, was chosen to be $0.5 \mathrm{~s}$. Finally, NOESY spectra of both (PTATBA and TBACl) solutions were measured in $\mathrm{D}_{2} \mathrm{O}$ under optimized conditions using a phase-sensitive NOESY pulse sequence ${ }^{36-38}$ (Bruker noesyph). The $90^{\circ}$ pulse duration was $14.5 \mu \mathrm{s}$. To build the complete NOESY spectra, 256 free induction decays (16 scans each) of 8192 pts were recorded with a time increment for the indirect dimension of $171 \mu \mathrm{s}$. A 2D Fourier transform with a zero filling to 1024 points in the $f_{1}$ domain was then applied to obtain the NOESY spectra. During the measurements, the temperature was kept at $298.1 \mathrm{~K}$ $( \pm 0.1 \mathrm{~K})$.

\section{SIMULATIONS}

Simulation Method. MD simulations of PTAA oligomers in the presence of different types of counterions neutralizing the charge of the oligomer were performed with the YASARA Structure. In these calculations, specificity of binding of alkali $\left(\mathrm{Li}^{+}, \mathrm{Na}^{+}\right.$, and $\left.\mathrm{Cs}^{+}\right)$and of tetramethylammonium $\left(\mathrm{TMA}^{+}\right)$, tetraethylammonium $\left(\mathrm{TEA}^{+}\right)$, tetrapropylammonium $\left(\mathrm{TPA}^{+}\right)$, and tetrabutylammonium $\left(\mathrm{TBA}^{+}\right)$ions to PTAA was investigated. In MD simulations, the AMBER14 force field under periodic boundary conditions and with explicit TIP3P water was used. Multiple time steps of $1.25 \mathrm{fs}$ for intramolecular and $2.5 \mathrm{fs}$ for intermolecular forces were used. In all cases, the initial configuration consisted of 4 copies of 13-mer PTAA being regularly distributed into a cubic box with a length of $70.91 \AA$ while initial positions of 52 copies of the corresponding counterion were randomly chosen. The remaining available space in the box was filled with TIP3P water molecules. The final density of water objects in the simulation box was $0.997 \mathrm{~g} \mathrm{~cm}^{-3}$. Thus, the number of water molecules in the cells differs slightly in accordance with the size of the counterion $\left(\mathrm{Li}^{+}: 11,460 ; \mathrm{Na}^{+}\right.$: 11,467; $\mathrm{Cs}^{+}: 11,467 ; \mathrm{TMA}^{+}: 11,380 ; \mathrm{TEA}^{+}: 11,284 ; \mathrm{TPA}^{+}$: 11,128 ; and $\left.\mathrm{TBA}^{+}: 10,980\right)$. An $8.0 \AA$ cutoff was used for Lennard-Jones forces. The particle mesh Ewald method was used to treat electrostatics. At the start of calculations, the energy of the system was minimized by simulated annealing; then, dynamics simulations were run at $298 \mathrm{~K}$. Temperature was adjusted using a Berendsen thermostat based on time-averaged temperature. The force field parameters for the PTAA oligomer and TAA ions $\left(\mathrm{TMA}^{+}, \mathrm{TEA}^{+}, \mathrm{TPA}^{+}\right.$, and $\left.\mathrm{TBA}^{+}\right)$were obtained with the multistep procedure YASARA-AutoSMILES. The procedure employed SMILES strings to identify known molecules (even if residue and atom names differ) and resorts to the AM1BCC and general AMBER force field approaches for all other molecules. AM1BCC charges were additionally improved by using known RESP (restrained electrostatic potential) charges of similar molecule fragments, again identified via SMILES strings. All together, $60 \mathrm{~ns}$ of MD was performed for each system.

The concentration used in simulations and in the computation of NOESY spectra $\left(c=0.2 \mathrm{~mol} \mathrm{~L}^{-1}\right)$ is higher than the experimental concentration $\left(c=0.02 \mathrm{~mol} \mathrm{~L}^{-1}\right)$. This relatively high concentration was used to reduce the number of water molecules included in simulations and therefore preventing unreasonably long simulation run times. On the other hand, the concentration in experiments was kept at 0.02 mol $\mathrm{L}^{-1}$, taking that most experimental data related to counterion binding and polyion conformation are available at this concentration. ${ }^{11}$

Calculation of NOESY Spectra. Following Macura and Ernst ${ }^{39}$ the computation of a NOESY spectrum requires the evaluation of the longitudinal magnetizations of groups of equivalent spins $M$ coupled by a relaxation matrix $\mathbf{R}$ 

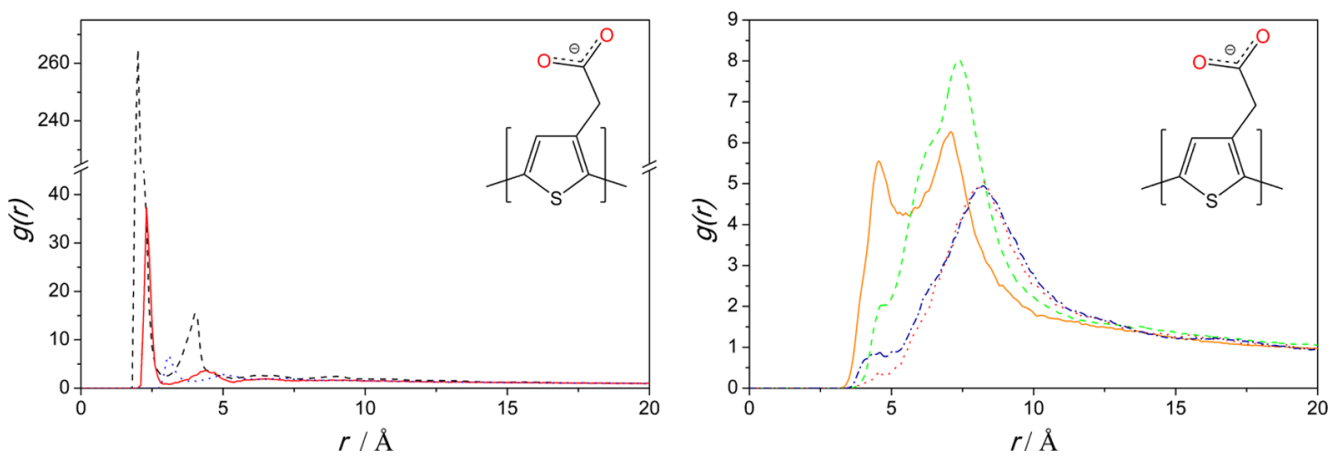

Figure 2. Left: Pair distribution function between oxygen atoms of the polyion (marked with red color in the inset of the figure) and alkali counterions $\left(\mathrm{Li}^{+}\right.$- black dashed line, $\mathrm{Na}^{+}$- red solid line, and $\mathrm{Cs}^{+}$- blue dotted line). Right: Pair distribution function between oxygen atoms of the polyion and the central nitrogen atom of TAA counterions $\left(\mathrm{TMA}^{+}\right.$— orange full line, $\mathrm{TEA}^{+}$— green dashed line, $\mathrm{TPA}^{+}$—red dotted line, and TBA ${ }^{+}$-blue dash-dot line).
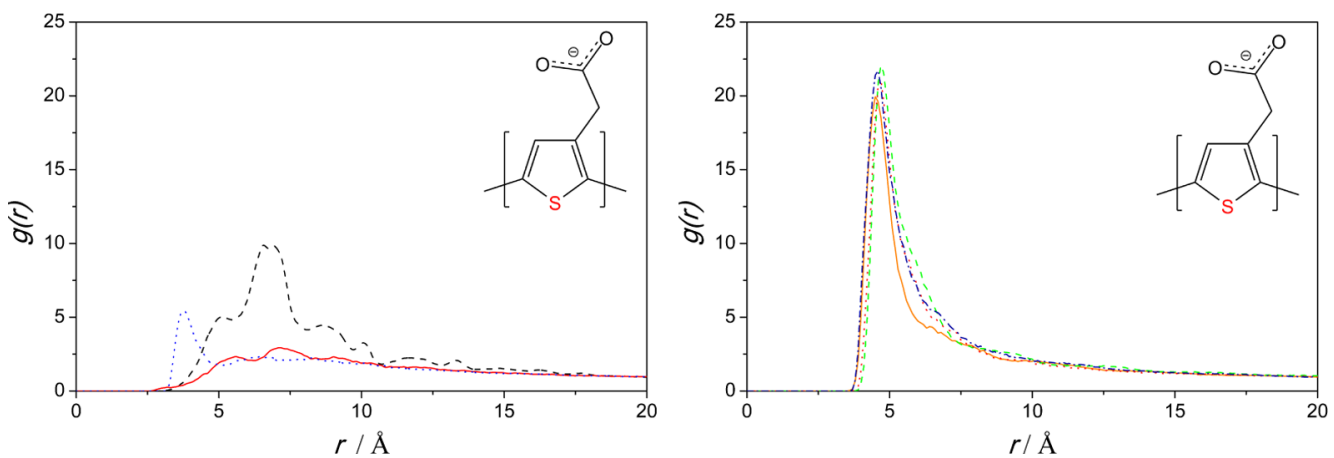

Figure 3. Left: Pair distribution function between sulphur atoms of the polyion (marked with red color in the inset of the figure) and alkali counterions ( $\mathrm{Li}^{+}$- black dashed line, $\mathrm{Na}^{+}$- red solid line, and $\mathrm{Cs}^{+}$- blue dotted line). Right: Pair distribution function between sulphur atoms of the polyion (marked with red color in this figure) and the central nitrogen atom of TAA counterions $\left(\mathrm{TMA}^{+}\right.$—orange full line, $\mathrm{TEA}^{+}$- $\mathrm{green}$ dashed line, $\mathrm{TPA}^{+}$red dotted line, and $\mathrm{TBA}^{+}$- blue dash-dot line).

$$
\frac{\mathrm{d} M}{\mathrm{~d} t_{\mathrm{m}}}+\mathbf{R} M=0
$$

where $t_{\mathrm{m}}$ is the mixing time. If the mixing time is set to zero, diagonal signal intensities are equal to signal intensities in the ${ }^{1} \mathrm{H}$ NMR spectrum, while all nondiagonal signal intensities are equal to zero. Following Peter et al., ${ }^{29}$ eq 1 is solved by diagonalizing the relaxation matrix $\mathbf{R}$.

The relaxation matrix $\mathbf{R}$ is built by considering the interaction between spin pairs and calculated from the elements of Solomon's matrix $\rho_{\mathrm{kk}}$ and $\sigma_{\mathrm{kl}}{ }^{40-42}$ coupling two $1 / 2$ spins $\mathrm{k}$ and 1 calculated as

$$
\begin{aligned}
& \rho_{\mathrm{kk}}=\frac{1}{10} K^{2}\left[3 J\left(2 \omega_{0}\right)+\frac{3}{2} J\left(\omega_{0}\right)+\frac{1}{2} J(0)\right] \\
& \sigma_{\mathrm{kl}}=\frac{1}{10} K^{2}\left[3 J\left(2 \omega_{0}\right)-\frac{1}{2} J(0)\right]
\end{aligned}
$$

where $J(\omega)$ is the spectral density, $\omega_{0}$ is the Larmor frequency, and $K$ is defined as

$$
K=\frac{\mu_{0} \hbar \gamma^{2}}{4 \pi}
$$

In the abovementioned equation, $\mu_{0}, \hbar$, and $\gamma$ are the vacuum permeability, Planck constant divided by $2 \pi$, and the gyromagnetic ratio of the proton, respectively.

According to the Wiener-Khinchin theorem, the spectral density is the Fourier transform of the correlation function of the magnetic noise felt by the nucleus under study, in other words, the dipole-dipole interaction fluctuation. The dipole-dipole interaction tensor $[\mathbf{D}(t)]$ writes in Cartesian coordinates as

$$
\mathbf{D}(t)=\frac{K}{r^{5}}\left(\begin{array}{lll}
3 x^{2}-r^{2} & 3 x y & 3 x z \\
3 y x & 3 y^{2}-r^{2} & 3 y z \\
3 z x & 3 z y & 3 z^{2}-r^{2}
\end{array}\right)
$$

As in ref 29, for all selected spin pairs, the dipole-dipole interaction tensor $\mathbf{D}(t)$ was computed at each time step from the simulated trajectories. In the second step, the correlation functions $C_{\alpha \beta}$ were computed for every element of the matrix $D_{\alpha \beta}$ and for every type of spin pairs as

$$
C_{\alpha \beta}(\tau)=\left\langle D_{\alpha \beta}(t) D_{\alpha \beta}(t+\tau)\right\rangle
$$

Finally, the autocorrelation function was calculated using eq 7.

$$
C(\tau)=\frac{1}{6} \sum_{\alpha, \beta=1}^{3, \beta \leq \alpha} C_{\alpha \beta}(\tau)
$$

The correlation function was then Fourier transformed to obtain the spectral density $J(\omega)$. For each spin pair, the spectral density $J(\omega)$ was evaluated at three frequencies: at zero frequency $(0 \mathrm{MHz})$, at the Larmor frequency $\left(\omega_{0}=500\right.$ $\mathrm{MHz})$, and at twice the Larmor frequency $\left(2 \omega_{0}=1000 \mathrm{MHz}\right)$ as needed for the evaluation of $\rho_{\mathrm{kk}}$ and $\sigma_{\mathrm{kl}}$ (eqs 2 and 3). 


\section{RESULTS AND DISCUSSION}

During previous studies, we studied aqueous solutions of PTAA using various experimental methods, being focused to the counterion-specific effects in these solutions. ${ }^{10,11,34,35,43}$

The experimentally determined degrees of bound alkali counterions agreed rather well ${ }^{11,35}$ both with the degree of condensed counterions within the frame of Manning's condensation theory ${ }^{4-46}$ and with the degree of electrostatically bound counterions as obtained from the cell model. ${ }^{47-49}$ Contrary to alkali counterions, for which their binding seems to be nonspecific, taking that it can be satisfactorily described by the abovementioned electrostatic theories, a higher degree of binding than that predicted by the electrostatic theories has been observed $^{11}$ for TAA (and especially for tetrabutylammonium) counterions in the given system. According to the obtained results, we concluded that the more extensive binding of $\mathrm{TBA}^{+}$ ions is the consequence of additional, presumably hydrophobic, ${ }^{50}$ interactions of $\mathrm{TBA}^{+}$with the PTAA main chain. To test this assumption, we performed MD simulations and measured NOESY spectra of PTATBA solutions as described in the following subsections.

Analysis of the Pair Distribution Functions: Specificity of the Binding Sites. One of the main aims of the present study was to establish whether the binding of a certain type of counterion is controlled through electrostatic or through hydrophobic interactions. For this purpose, the radial distribution functions (RDFs; less specialized readers can find some basic additional information about RDFs in the Supporting Information) between atoms in the polyion chain and counterions were computed from MD simulations (Figures 2 and 3). To assess the binding of counterions to the hydrophilic moiety of the polyelectrolyte chain, the RDFs between the centers of counterions and oxygen atoms of the carboxylate group were analyzed. The tendency of counterions to bind to the hydrophobic moiety was evaluated from the value of the $\mathrm{RDF}$ at the contact distance between counterions and sulphur atoms of PTAA thiophene ring.

In Figure 2, the RDFs $g_{\mathrm{xo}}(r)$ between the center of counterions $\mathrm{X}^{+}$and oxygen atoms of PTAA are plotted. The most intense peak for alkali counterions (Figure 2, left), occurring around $2.25 \AA$, belongs to lithium counterions. The most intense peak in the RDF of sodium ions is notably lower and positioned at a slightly larger distance from oxygen atoms (at around $2.5 \AA$ ) than the peak for $\mathrm{Li}^{+}$ions. The first peak in the $\mathrm{RDF}$ of $\mathrm{Cs}^{+}$is very weakly expressed and can be found at a distance of around $3 \AA$. These three distances correspond to the distances between the center of the oxygen atom and centers of the given three alkali counterions when they are in the direct contact (formation of contact ion pairs). All these RDFs show also further (second, third, ...) and less sharply expressed peaks, but these peaks are, rather than being part of the second layer of counterions, the consequence of the oligoion structure. More careful inspection showed that the position of the second peak corresponds to the distance between the counterion, bound on the carboxylate group, and oxygen atom of the carboxylate group from the adjacent monomeric unit.

The information that could be additionally elucidated from this plot (Figure 2, left) is also the relative extent of binding of a certain type of the counterion when compared to others. Taking into account the intensity of peaks and their position in RDFs, one could then expect that the degree of bound $\mathrm{Li}^{+}$ions would be the highest and the one of $\mathrm{Cs}^{+}$, the lowest. These results of
MD simulations contradict our previous experimental studies $^{11,35}$ showing that the degree of bound alkali counterions is practically the same for all these counterions and that this degree of bound alkali counterions is determined by electrostatic interactions. ${ }^{43}$ The discussion about the possible reason for this discrepancy for the extent of ion binding will take place at the end of this subsection.

Considering the more hydrophobic character of TAA counterions, one may expect that their distribution around the PTAA polyion will differ from the one of alkali ions. Indeed, contrary to alkali counterions where more or less sharp first peak was observed in all $g_{\mathrm{XO}}(r)$, in the case of TAA counterions, peaks are broader (with the exception of $\mathrm{TMA}^{+}$ions) and located (Figure 2, right) at larger distances from the oxygen atom (7$8.5 \AA$ ). Regarding these distances, the partial exception is again $\mathrm{TMA}^{+}$ions where the first (and little less intensive than the second one) peak occurs at the distance slightly less than $5 \AA$ from oxygen atoms. This last distance indicates that $\mathrm{TMA}^{+}$ counterions may be found with a noticeable probability also in very close proximity to the carboxylate group. The appearance of main peaks in RDFs of other TAA ions $\left(\mathrm{TEA}^{+}-\mathrm{TBA}^{+}\right)$and of the second peak in the RDF of $\mathrm{TMA}^{+}$counterions is not due to the direct binding of these ions to the carboxylate group but to their binding to thiophene rings. This statement was confirmed also through surveying RDFs $g_{X S}(r)$ between sulphur atoms of PTAA and counterions.

The inspection of RDFs between sulphur atoms of PTAA and alkali counterions (Figure 3, left) reveals no accumulation of alkali counterions in close proximity of sulphur atoms. Instead, the RDFs exhibit rather broad peaks with shoulders located at larger distances. The only relatively sharp peaks that exist in these $\mathrm{RDFs}$ is the peak at approximately $6.5 \AA$ belonging to $\mathrm{Li}^{+}$ counterions and the peak at approximately $3.75 \AA$ belonging to $\mathrm{Cs}^{+}$counterions. The latter peak indicates some minor degree of binding of $\mathrm{Cs}^{+}$in the vicinity of the PTAA aromatic ring. The peak in the $\mathrm{Li}^{+} \mathrm{RDF}$ at a distance of approximately $6.5 \AA$ as well as the broad peak for $\mathrm{Na}^{+}$and the shoulder for $\mathrm{Cs}^{+}$ions at this distance actually confirms the existence of site binding (direct contact) of alkali ions to oxygen atoms from PTAA carboxylate groups, as already indicated by $g_{\mathrm{xo}}(r)$. Shoulders are observed because of the different conformations of the oligoion side chains while the slow decrease in the $g_{\mathrm{XS}}(r)$ value at large distances may be observed because of the binding of alkali counterions to carboxylate groups of adjacent monomeric units.

Here, for all TAA counterions, a sharp peak may be observed at a distance of approximately $5 \AA$ (Figure 3 , right). The peak height and position seem not to be dependent much on the size of TAA counterions. This peak testifies that bound TAA counterions are site-bound on the hydrophobic part of the polyelectrolyte. Taking into account the fact that the alkyl chains of TAA counterions are hydrophobic as well, we may infer that the binding position of TAA counterions is determined by hydrophobic interactions. The results of simulations therefore suggest that the binding of counterions to oligoions is at least partially driven by hydrophobic interactions.

Surprisingly, the correlation peak height does not increase with increasing TAA counterion size. The relative tendency of TAA counterions to bind to the polyion in the vicinity of sulphur atoms can be estimated from the integration of the pair distribution function over the spherical volume encompassing sulphur atoms. As can be seen from Figure 3 (right), the values of these integrals would not change significantly (and together with them also the degree of bound counterions) with the 


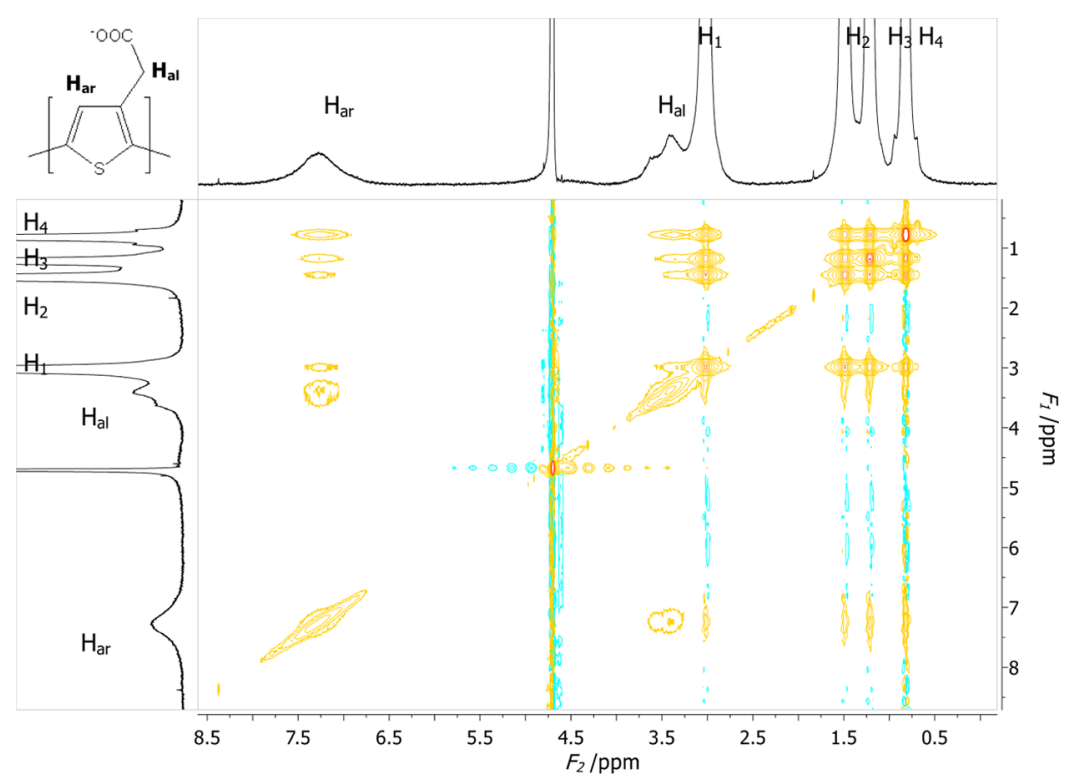

Figure 4. NOESY spectrum of $0.02 \mathrm{~mol} \mathrm{~L}^{-1}$ solution of PTATBA in $\mathrm{D}_{2} \mathrm{O}$. Blue represents negative peaks, while yellow and red represent positive peaks. The signal at $4.7 \mathrm{ppm}$ is the residual water peak. $t_{\mathrm{m}}=0.5 \mathrm{~s}$.

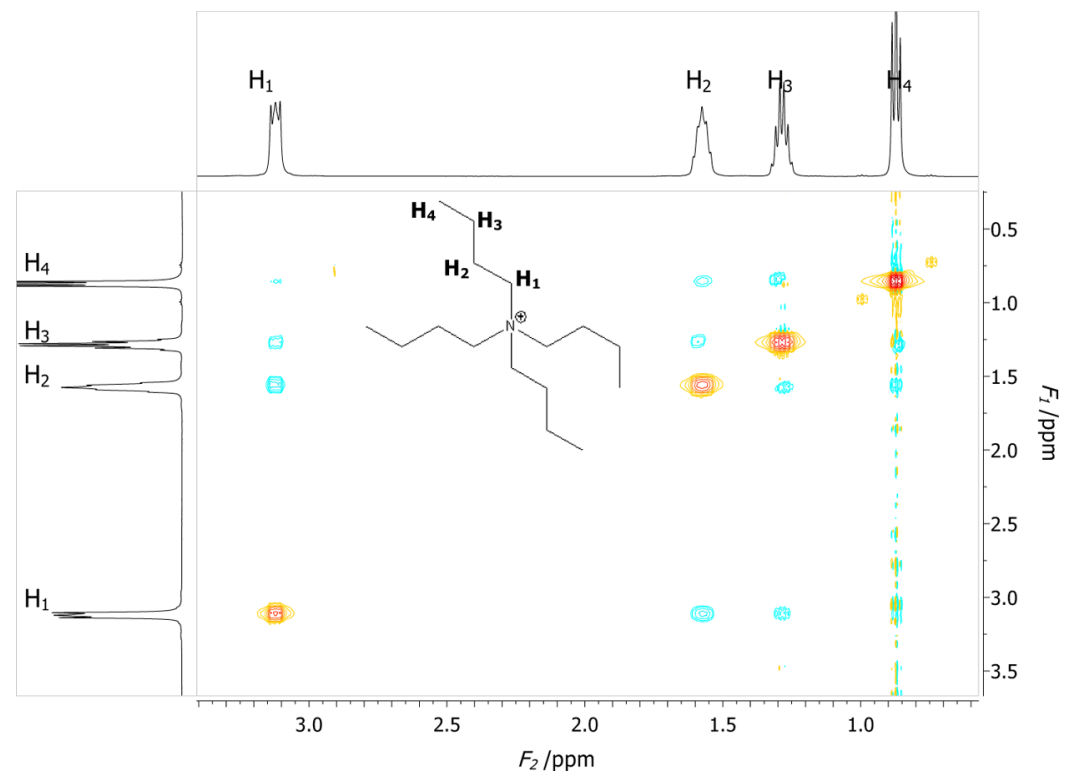

Figure 5. NOESY spectrum of $0.02 \mathrm{~mol} \mathrm{~L}^{-1}$ solution of $\mathrm{TBACl}$ in heavy water. The color scale is the same as in Figure $4 t_{\mathrm{m}}=0.5 \mathrm{~s}$.

increasing size of TAA counterions. This result appears in contradiction with the experimental evidence that the degree of bound TAA counterions increases with the size of the counterion. $^{11}$ As already mentioned before, ranging of the degree of alkali counterions bound to carboxylic groups as derived from MD simulations (Figure 2-left) is questionable. When this observation is coupled with the ranging of the degree of alkali counterions bound to thiophene groups (Figure 3left), one can claim that also the theoretically predicted tendency of alkali counterions to bind to PTAA is doubtful when quantitative forecast is considered. Although experiments clearly show that $\mathrm{Li}^{+}, \mathrm{Na}^{+}$, and $\mathrm{Cs}^{+}$ions are within the experimental error to the same extent bound to PTAA, ${ }^{11,35} \mathrm{MD}$ simulations definitely forecast the trend of diminishing binding with the increase in the size of alkali counterions $\left(\mathrm{Li}^{+}>\mathrm{Na}^{+}>\mathrm{Cs}^{+}\right)$.

We ascribe the weaknesses of the MD simulations to the limit of the force field used. Namely, it is known that classical nonpolarizable water models that neglect explicit polarization have limited predicting capabilities when they are used for studying hydration in strong electrostatic fields. ${ }^{51}$ Moreover, also MD simulations of concentrated aqueous solutions of ordinary salts give only a poor fit to the experimental data when standard nonpolarizable force fields are used. ${ }^{52,53}$ The predictive power of such MD simulations could be improved if polarization effect is taken into account. However, the use of polarizable force fields significantly increases the simulation time, and the development and optimization of such force fields lies beyond the scope of this study. Consequently, also in the case when polarization effect would be taken into account, this would not be definite proof that TAA counterions are bound to the hydrophobic moiety of PTAA. In order to verify the hypothesis of binding of larger TAA counterions to the thiophene ring, the experimental NOESY spectrum of PTATBA was measured and 


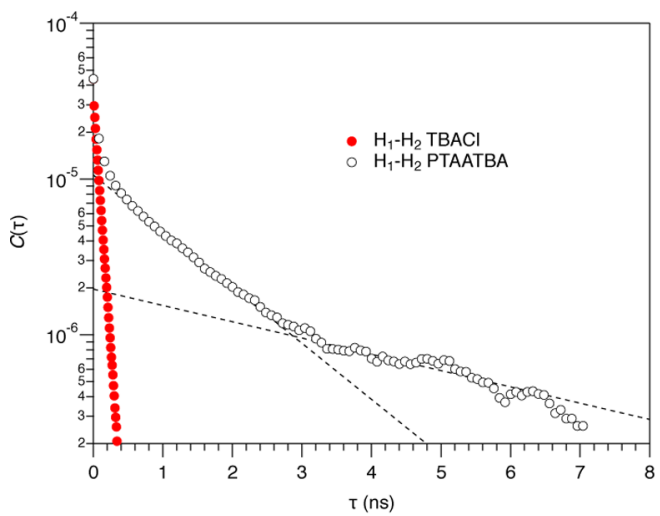

(a)

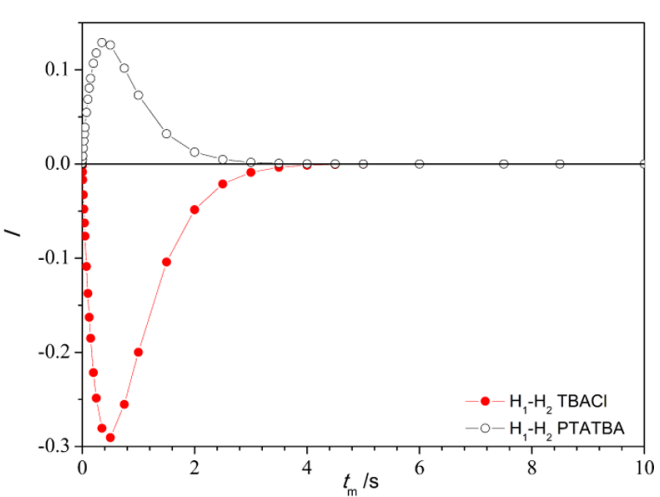

(b)

Figure 6. Autocorrelation function $\mathrm{C}(\tau)$ as a function of correlation time $\tau$ for interactions between $\mathrm{H}_{1}$ and $\mathrm{H}_{2}$ type of hydrogen atoms in solution of $\mathrm{TBACl}$ (filled red circles) and PTATBA (hollow black circles) (a). It can be clearly seen that $C(\tau)$ decays significantly faster in the case of TBACl solution than in the case of PTATBA solution. In (b), the corresponding buildup curves are represented.

compared to the simulated NOESY spectrum as derived from MD simulations.

Measured NOESY Spectra. In Figure 4, the NOESY spectrum of PTATBA in heavy water is shown $\left(c_{\mathrm{p}}=0.02 \mathrm{~mol}\right.$ $\mathrm{L}^{-1}, T=298.15 \mathrm{~K}$ ). As in any NOESY spectrum, two types of peaks are present-diagonal peaks and cross-peaks. As already discussed, the appearance of cross-peaks is the consequence of interactions between magnetic dipoles of different hydrogen nuclei. ${ }^{42}$

In the spectrum shown in Figure 4, we may observe the following cross-peaks belonging to PTATBA:

- between aromatic $\left(\mathrm{H}_{\mathrm{ar}}\right)$ and aliphatic $\left(\mathrm{H}_{\mathrm{al}}\right)$ hydrogen atoms of the polyion,

- between all four types of hydrogen atoms $\left(\mathrm{H}_{1}-\mathrm{H}_{4}\right)$ of tetrabutylammonium counterions, and

- between each of both types of hydrogen atoms of the polyion $\left(\mathrm{H}_{\mathrm{ar}}\right.$ and $\left.\mathrm{H}_{\mathrm{al}}\right)$ and each of all four types of hydrogen atoms of tetrabutylammonium counterions $\left(\mathrm{H}_{1}-\mathrm{H}_{4}\right)$.

The existence of cross-peaks confirms that magnetization transfer is possible and that proximity, even transient, between the different protons is involved. The sign of the cross-peaks in NOESY spectra reveals important dynamic information. As can be seen from eq 3 , it only depends on the values of the spectral density $J(\omega)$ at twice the Larmor frequency $\left(\omega=2 \omega_{0}\right)$ and at zero frequency $(\omega=0)$. For fast motions compared to the inverse Larmor frequency $\left(\omega_{0} \tau_{\mathrm{c}} \ll 1\right.$, where $\tau_{\mathrm{c}}$ is the correlation time of the motion), we are in the extreme narrowing case where $J\left(2 \omega_{0}\right) \simeq J(0)$ and NOESY cross-peaks are negative (opposite to the sign of diagonal peaks). ${ }^{42}$ For the case of slow dynamics, $J\left(2 \omega_{0}\right) \ll J(0)$, the signs of $\sigma_{\mathrm{kl}}$ are changed, and consequently, the rise of the corresponding positive cross-peaks can be observed. It is possible to see from Figure 4 that all cross-peaks in the NOESY spectrum of PTATBA solution are positive.

The dynamics of the polyion is, due to its high molar mass, slow, and the sign of the cross-peaks between aromatic and aliphatic protons are indeed expected to be positive.

On the other hand, for TBA hydrogen atoms $\mathrm{H}_{1}$ to $\mathrm{H}_{4}$ in aqueous solution of pure $\mathrm{TBACl}$, the expected sign of intramolecular cross-peaks would be, due to the smaller molar mass of $\mathrm{TBA}^{+}$salt and accordingly fast dynamics of $\mathrm{TBA}^{+}$ions, negative. This assumption is confirmed by the experimental
NOESY spectrum of TBACl in $\mathrm{D}_{2} \mathrm{O}$ recorded at the same concentration $\left(0.02 \mathrm{~mol} \mathrm{~L}^{-1}\right)$ and shown in Figure 5.

However, in the presence of PTAA, both the intramolecular cross-peaks of $\mathrm{TBA}^{+}$and the intermolecular cross-peaks between $\mathrm{TBA}^{+}$and PTAA are positive (Figure 4). We can then infer that when the $\mathrm{TBA}^{+}$counterion binds to PTAA polyions its dynamics is sufficiently damped, very likely because of strong binding, to reverse the sign of negative peaks found in the TBACl NOESY spectrum. This finding is in good agreement with a high degree of binding of $\mathrm{TBA}^{+}$counterions, observed in our previous study. ${ }^{11}$

Calculated NOESY Spectrum and Comparison. NOESY spectra have been calculated from MD simulation of 4 oligoions with a length of 13 monomeric units and $52 \mathrm{TBA}^{+}$counterions, following the procedure described in the section Calculation of NOESY spectra. Before discussing the output of the computation, namely, the calculated NOESY spectra, it is interesting to take a look at the correlation function that gives a more direct access to the change of dynamics. As an instructive example, the $\mathrm{H}_{1}-\mathrm{H}_{2}$ correlation function both in the presence and in the absence of the PTAA polyelectrolyte is plotted in Figure 6. The decay of the correlation function in TBACl solution is, as expected, very fast showing an exponential decay (linear in a log-lin plot) having the correlation time smaller than $1 \mathrm{~ns}$, consistent with the data of ref 54. In the presence of PTAA, the decay of the correlation function is considerably slowed down and exhibits different regimes. At very short times, the first nonexponential relaxation is observed and this one can be ascribed to the internal motion of the alkyl moieties of nonbound $\mathrm{TBA}^{+}$ions. This very fast regime will not be discussed further because the characteristic times are too short to have a significant effect on the NOESY spectrum. At longer correlation times, two distinct exponential decays can be identified. Although it is not possible to assign these decays to specific motions straightforwardly, one can infer that the slowed down tumbling of the counterion on the polyelectrolyte chain and the slow motion of the polyelectrolyte chain itself can be ascribed to these two decays.

A practical issue is the difficulty to compute numerically and accurately the Fourier transform of the correlation function in the case of slow relaxation. Indeed, the slow component is noisy and the statistics is poor because of the finite duration of the simulation runs (as seen in Figure 6). Consequently, the correlation functions were fitted and the spectral densities were 

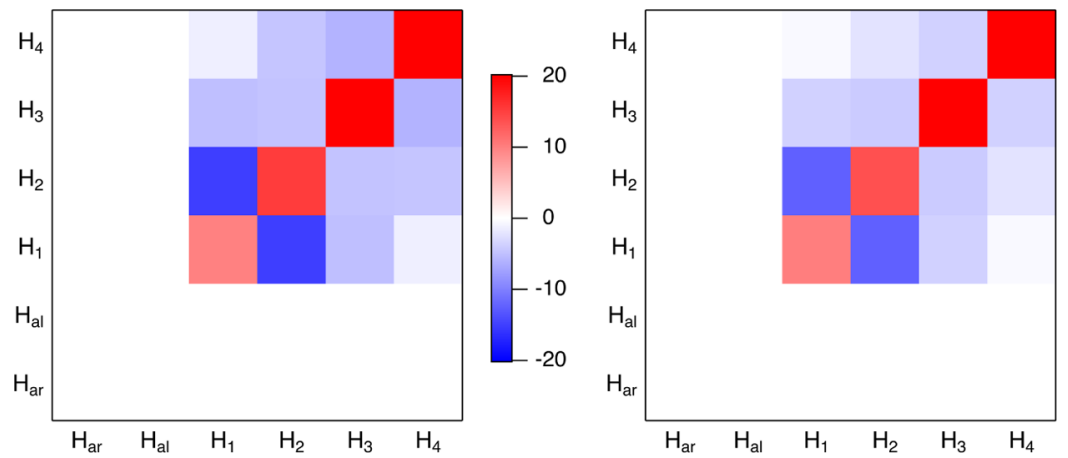

Figure 7. Simplified NOESY spectra $\left(t_{\mathrm{m}}=0.5 \mathrm{~s}\right)$ for a solution of TBACl in $\mathrm{D}_{2} \mathrm{O}$. Left: Experimental NOESY integrals, $\left(c=0.02 \mathrm{~mol} \mathrm{~L}{ }^{-1}\right)$. Right: Computed NOESY integrals, $\left(c=0.2 \mathrm{~mol} \mathrm{~L}^{-1}\right)$.
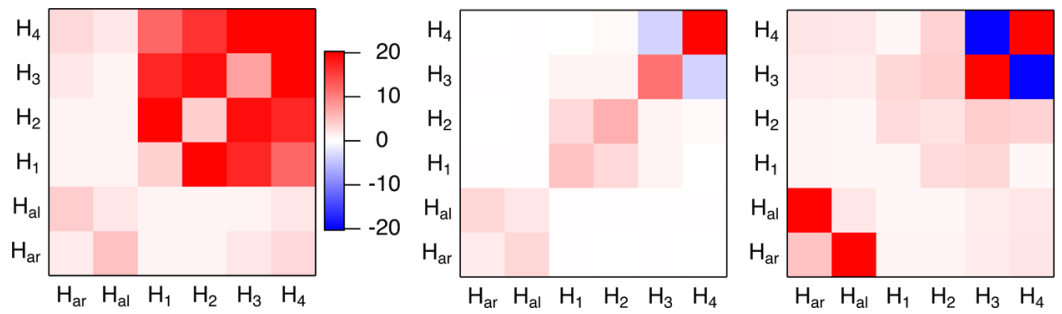

Figure 8. Simplified NOESY spectra for the solution of PTATBA in $\mathrm{D}_{2} \mathrm{O}$. Left: Integrals obtained from the experimental NOESY spectrum, $\left(t_{\mathrm{m}}=0.5 \mathrm{~s}\right.$, $\left.c=0.02 \mathrm{~mol} \mathrm{~L}^{-1}\right)$. Middle: Computed NOESY integrals, $\left(t_{\mathrm{m}}=0.5 \mathrm{~s}, c=0.2 \mathrm{~mol} \mathrm{~L}^{-1}\right)$. Right: Computed NOESY integrals, $\left(t_{\mathrm{m}}=2.0 \mathrm{~s}, c=0.2 \mathrm{~mol} \mathrm{~L}-1\right)$.

computed with the analytical Fourier transform containing the fitted parameters. For the aqueous TBACl solutions, a monoexponential decay was used, whereas for PTATBA solutions, the correlation functions $C(\tau)$ were fitted with the sum of two exponential decays.

From the correlation functions, the relaxation matrix $\mathbf{R}$ and the evolution of the magnetization were computed. The socalled NOE buildup curves, displaying the signal intensity (I) versus mixing time $\left(t_{\mathrm{m}}\right)$, were then calculated and are reported in the Supporting Information. In the case of diagonal peaks, they straightforwardly represent the longitudinal relaxation ${ }^{39,42}$ of the different types of protons. For the cross-peaks, they show the exchange of magnetization between the different types of protons during the mixing delay. Starting from zero at $t_{\mathrm{m}}=0$, the intensity of cross-peaks increases (or decreases) because of magnetization exchange and it finally decreases because of the longitudinal relaxation.

Because the complete calculation of NOE spectra would require the assignment of resonance frequencies (chemical shifts), couplings, and peak widths, we decided to use a simplified representation to compare directly the experimental data and the MD results for the same mixing time $t_{\mathrm{m}}$. The results are plotted in Figures 7 and 8, where both the experimental intensities (integrated peaks) and the computed intensities are reported. To allow a direct comparison, the results were normalized, and because of their much larger amplitude, diagonal peaks were divided by the factor of 10 .

In Figure 7, the simplified NOESY spectra at $t_{\mathrm{m}}=0.5 \mathrm{~s}$ for a solution of TBACl in $\mathrm{D}_{2} \mathrm{O}$ are reported. A surprisingly good agreement between the experimental data and the $\mathrm{MD}$ simulation results can be seen. The signals of cross-peaks in simulated $\mathrm{TBACl}$ solutions are all negative (are of the opposite sign to the ones of diagonal peaks) which are in quantitative agreement with the experimental TBACl NOESY spectrum, indicating a fast dynamics of the ion. $\mathrm{H}_{1}$ protons display the shortest $T_{1}$ and thus the largest attenuation among the diagonal peaks, as expected for the most rigid part of the $\mathrm{TBA}^{+}$ion. ${ }^{54}$ The dipolar interactions in this solution are mainly intramolecular and their intensities steeply decrease with the distance between protons, that is, the strongest interactions are expected to occur between vicinal protons. These good results validate the method used to compute the NOESY spectra.

In Figure 8, the same approach was used for the comparison of the experimental and calculated NOESY spectra of PTATBA solution in $\mathrm{D}_{2} \mathrm{O}$. This system is more complex and larger differences between the measured and calculated NOESY spectrum may be expected. Following the discussion about the computed RDFs (see the section Analysis of the Pair Distribution Functions), one source of discrepancy between the experimental and calculated NOESY spectrum may be the underestimated degree of bound $\mathrm{TBA}^{+}$ions. Consequently, such a simulated spectrum should be more strongly influenced by the characteristics of fast dynamics of free (i.e., nonbound) $\mathrm{TBA}^{+}$ ions than that of the experimental one. Because of the overrepresentation of free $\mathrm{TBA}^{+}$ions in the PTATBA $\mathrm{MD}$ simulations, the transfer of magnetization leading to the occurrence of both inter- and intramolecular cross-peaks involving protons from $\mathrm{TBA}^{+}$ions is expected to be underestimated in the calculated NOESY spectrum. In other words, because of on average larger separation distances between the polyelectrolyte and the counterion, the computed crossrelaxation rate $\sigma_{\mathrm{kl}}$ (eq 3 ) is smaller leading not only to lower amplitudes for the cross-peaks but also to a delay of the maximum value of the cross-peak in terms of mixing time. In order to account for this possibility shown in Figure 8, the calculated NOESY spectra are shown for $t_{\mathrm{m}}=0.5 \mathrm{~s}$ and for $t_{\mathrm{m}}=$ $2.0 \mathrm{~s}$.

The PTAA signals show a positive intramolecular cross-peak between $\mathrm{H}_{\mathrm{al}}$ and $\mathrm{H}_{\mathrm{ar}}$ which is an expected signature of the slow dynamics of the polyion chain. Regarding this peak, the resemblance between the measured and calculated spectrum at $t_{\mathrm{m}}=0.5 \mathrm{~s}$ is quite satisfactory thus confirming reasonably good 
choice of MD parameters for the simulation of the PTAA polyion itself.

The effect of binding of counterions is clearly visible both in the experimental spectra and in the calculated spectra. The diagonal peaks are smaller corresponding to the faster longitudinal relaxation, and the counterion cross-peaks are all positive (apart from the $\mathrm{H}_{3}-\mathrm{H}_{4}$ cross-peak in the computed spectra). These two observations can be ascribed to the slowed down dynamics because of the significant binding of $\mathrm{TBA}^{+}$ions to the polyion. ${ }^{11}$ Similarly, as for $\mathrm{TBA}^{+}$ions in $\mathrm{TBACl}$ solutions, the absolute amplitude of $\mathrm{TBA}^{+}$cross-peaks in PTATBA solution decreases with the distance between the protons. MD simulation predicts faster dynamics of the $\mathrm{H}_{3}-\mathrm{H}_{4}$ dipole-dipole interaction leading to a negative calculated peak (both for $t_{\mathrm{m}}=$ $0.5 \mathrm{~s}$ and $\left.t_{\mathrm{m}}=2.0 \mathrm{~s}\right)$. On the contrary, the experimental spectrum shows a strong slowdown of dynamics of the $\mathrm{H}_{3}-\mathrm{H}_{4}$ interaction as can be seen by the positive $\mathrm{H}_{3}-\mathrm{H}_{4}$ cross-peak. Such a feature can be ascribed to the specific attractive interaction between the methyl group and hydrophobic part of the polyion.

Considering that $\mathrm{TBA}^{+}$cross-peaks in the experimental and calculated spectra of PTATBA solution differ notably, one has to comment these differences further. The signs of all cross-peaks induced by the interactions between the protons of the counterion and of the polyion are positive, indicating a slow dynamics (see Figures S5 and S6). The cross-peak intensities in the simulated NOESY spectrum at $t_{\mathrm{m}}=0.5 \mathrm{~s}$ show only a qualitative agreement with the ones in the experimental spectrum (calculated intensities are around 6 times smaller than the measured ones)-see the left and the middle graphs shown in Figure 8. However, this agreement is greatly increased if one allows longer mixing times for the transfer of magnetization via cross-relaxation in the calculated NOESY spectrum. In this case, for $t_{\mathrm{m}}=2.0 \mathrm{~s}$, a nearly quantitative agreement of intermolecular cross-peak intensities in the experimental and calculated NOESY spectrum can be observed (the left and right graph shown in Figure 8).

The part of the calculated NOESY spectrum (both for $t_{\mathrm{m}}=0.5$ $\mathrm{s}$ and $t_{\mathrm{m}}=2.0 \mathrm{~s}$ ) that differs the most from the measured NOESY spectrum is the intramolecular cross-peaks of $\mathrm{TBA}^{+}$counterions (the most exposed case is the $\mathrm{H}_{3}-\mathrm{H}_{4}$ peak). One can safely assume that a higher degree of bound $\mathrm{TBA}^{+}$ions than that detected by the MD simulations would make intramolecular $\mathrm{TBA}^{+}$cross-peaks more positive because of experienced sloweddown dynamics of $\mathrm{TBA}^{+}$ions. Failure of the MD simulations to accurately describe intermolecular interactions between $\mathrm{TBA}^{+}$ counterions and the PTAA polyion may stem from two reasons. First, as it was already discussed when commenting RDFs between alkali counterions and the polyion, the used MD simulations predicted different degrees of bound counterions for alkali counterions while two different experimental techniques showed that the degree of bound alkali counterions is essentially the same for all these counterions. ${ }^{11,35}$ Second, the accuracy of the modeling of water properties in complex aqueous systems by $\mathrm{MD}$ is still to improve. ${ }^{55-57}$ In this regard, the most striking difference between the experimental and computed NOESY spectra (i.e., the sign of the $\mathrm{H}_{3}-\mathrm{H}_{4}$ cross-peak) may also be attributed to the imperfect ability of the MD simulation to deal correctly with hydrophobic interactions in the studied system.

At the end, we may stress one more time that the extent of binding was underestimated by the MD simulation. This is not unexpected if we consider that hydrophobic interactions contribute significantly to a high degree of binding in the experimental PTATBA system ${ }^{11}$ and that these interactions are difficult to simulate correctly with nonpolarizable models of water. ${ }^{58}$ It is known that additive force fields are a limiting factor in the accuracy of MD simulations when the simulated system consists both of highly polar and hydrophobic environments. ${ }^{59}$ The use of more advanced water models that include polarization effects or alternatively following one of the approaches that improve accuracy of additive force fields (e.g., optimization of the pairwise Lennard-Jones interaction parameters) would most probably result in a better agreement between the experimental and calculated NOESY spectra but it would require also notably longer computational times. Furthermore, because of the limited time range accessible to MD simulations, the current method cannot describe entirely the slow dynamics of the polyion and the associated counterion dynamics that are also probed by NOESY. Finally, the effect of the polydispersity in size of the polyelectrolyte is difficult to take into account in the simulations because of the limited size of the system. This polydispersity assuredly results in a polydispersity of dynamics that modifies the intensities of the NOESY peaks. In spite of these deficiencies, the dynamics of bound counterions from the MD simulations still seems to be sufficiently attenuated that the sign of almost all cross-peaks is positive which agrees fairly well with the experimental NOESY spectrum.

\section{CONCLUSIONS}

To investigate the specificity of binding of ions onto a conjugated polyelectrolyte, PTAA, we use a combination of MD simulations and NMR spectroscopy, especially NOESY experiments. The analysis of RDFs, obtained from MD simulation trajectories, showed a marked specificity for the location of binding that differs for alkali counterions which bind in the vicinity of the PTAA carboxylate group and TAA counterions which bind in the vicinity of the thiophene ring of the main polyion chain. In addition, only the smallest TAA ions, that is, tetramethylammonium, are capable of binding to the PTAA carboxylate group in a similar way to that of alkali ions. However, the quality of the force field used in the MD simulation is improvable because the computed degree of bound $\mathrm{TBA}^{+}$ions to the PTAA polyion is lower than that of the experimentally determined one. ${ }^{11}$ Nevertheless, such improvements would impose significantly higher computational costs or would require further studies of its own.

The NOESY spectra both of TBACl and of PTATBA solutions were measured and compared to intensities of NOESY spectra calculated for these solutions from MD simulations. Although the calculated NOESY spectrum of TBACl solution agrees remarkably well with that of the measured one, the agreement is only partial in the case of PTATBA solution. As expected, the polyelectrolyte internal dynamics is found to be slow. The presence of PTAA modifies the internal dynamics of $\mathrm{TBA}^{+}$ions, which shows a distinct overall slowdown upon binding, even if the dynamics of the methyl groups fails to be captured faithfully by the MD simulation. Both the computed and experimental NOESY spectra indicate a preferential binding of $\mathrm{TBA}^{+}$ions in the vicinity of the hydrophobic moiety of the polyelectrolyte. However, because of the lower degree of binding of the counterion predicted by the simulations and evidenced from the computed RDFs, longer mixing times than those for the experiment are needed to obtain comparable features in the computed NOESY spectrum. 


\section{ASSOCIATED CONTENT}

\section{(s) Supporting Information}

The Supporting Information is available free of charge at https://pubs.acs.org/doi/10.1021/acs.macromol.9b02161.

Computed buildup curves and basic information about the meaning of RDFs (PDF)

\section{AUTHOR INFORMATION}

\section{Corresponding Author}

Janez Cerar - Faculty of Chemistry and Chemical Technology,

University of Ljubljana, SI-1000 Ljubljana, Slovenia;

○ orcid.org/0000-0002-4354-4783; Email: janez.cerar@

fkkt.uni-lj.si

\section{Authors}

Gregor Hostnik - Faculty of Chemistry and Chemical Technology, University of Ljubljana, SI-1000 Ljubljana, Slovenia; Faculty of Chemistry and Chemical Technology, University of Maribor, SI-2000 Maribor, Slovenia; (ㄱ) orcid.org/ 0000-0002-7570-7460

Crtomir Podlipnik - Faculty of Chemistry and Chemical Technology, University of Ljubljana, SI-1000 Ljubljana, Slovenia Guillaume Mériguet - Sorbonne Université, CNRS, Laboratoire PHENIX, F-75005 Paris, France

Complete contact information is available at:

https://pubs.acs.org/10.1021/acs.macromol.9b02161

\section{Notes}

The authors declare no competing financial interest.

\section{ACKNOWLEDGMENTS}

This study was supported by the Slovenian Research Agency fund (ARRS) through the Program 0103-0201. The use of instruments at the NMR Spectroscopy Unit of Research Infrastructure center UL FCCT in performing preliminary measurements is acknowledged. G.H. was supported by the National Grant for young researchers, Slovenian Ministry of Education, Science and Sports, and the European Regional Development Fund through operation C3330-17-529001. Prof. Dr. Vojko Vlachy (University of Ljubljana) is acknowledged for valuable discussion through this work. The authors warmly thank Prof. Bernard Ancian for his insights and fruitful advice about the NOE and its applications. Dr. Isabelle Correia (Laboratoire des Biomolécules, Sorbonne University) is thanked for the access to the $500 \mathrm{MHz}$ spectrometer. We are really grateful to Dr. Natalie Malikova for her comments and suggestions about the manuscript. The authors would like to acknowledge networking support by the COST Action CA15209 (EURELAX).

\section{REFERENCES}

(1) Béra-Abérem, M.; Ho, H.-A.; Leclerc, M. Functional polythiophenes as optical chemo- and biosensors. Tetrahedron 2004, 60, 11169-11173.

(2) Cheng, Y.-J.; Yang, S.-H.; Hsu, C.-S. Synthesis of Conjugated Polymers for Organic Solar Cell Applications. Chem. Rev. 2009, 109, 5868-5923.

(3) Liu, X.; Fan, Q.; Huang, W. DNA biosensors based on watersoluble conjugated polymers. Biosens. Bioelectron. 2011, 26, 21542164 .

(4) He, Z.; Zhong, C.; Huang, X.; Wong, W.-Y.; Wu, H.; Chen, L.; Su, S.; Cao, Y. Simultaneous Enhancement of Open-Circuit Voltage,
Short-Circuit Current Density, and Fill Factor in Polymer Solar Cells. Adv. Mater. 2011, 23, 4636-4643.

(5) Chang, Y.-M.; Zhu, R.; Richard, E.; Chen, C.-C.; Li, G.; Yang, Y. Electrostatic Self-Assembly Conjugated Polyelectrolyte-Surfactant Complex as an Interlayer for High Performance Polymer Solar Cells. Adv. Funct. Mater. 2012, 22, 3284-3289.

(6) Wang, H.-J.; Chen, C.-P.; Jeng, R.-J. Polythiophenes Comprising Conjugated Pendants for Polymer Solar Cells: A Review. Materials 2014, 7, 2411-2439.

(7) Muthukumar, M. 50th Anniversary Perspective: A Perspective on Polyelectrolyte Solutions. Macromolecules 2017, 50, 9528-9560.

(8) McCullough, R. D.; Ewbank, P. C.; Loewe, R. S. Self-Assembly and Disassembly of Regioregular, Water Soluble Polythiophenes: Chemoselective Ionchromatic Sensing in Water. J. Am. Chem. Soc. 1997, 119, 633-634.

(9) Kim, B.; Chen, L.; Gong, J.; Osada, Y. Titration Behavior and Spectral Transitions of Water-Soluble Polythiophene Carboxylic Acids. Macromolecules 1999, 32, 3964-3969.

(10) Hostnik, G.; Vlachy, V.; Bondarev, D.; Vohlídal, J.; Cerar, J. Saltspecific effects observed in calorimetric studies of alkali and tetraalkylammonium salt solutions of poly(thiophen-3-ylacetic acid). Phys. Chem. Chem. Phys. 2015, 17, 2475-2483.

(11) Hostnik, G.; Bončina, M.; Dolce, C.; Mériguet, G.; Rollet, A.-L.; Cerar, J. Influence of counterions on the conformation of conjugated polyelectrolytes: the case of poly(thiophen-3-ylacetic acid). Phys. Chem. Chem. Phys. 2016, 18, 25036-25047.

(12) Semenyuk, P. I.; Zhiryakova, M. V.; Izumrudov, V. A. Supercharged Polyplexes: Full-Atom Molecular Dynamics Simulations and Experimental Study. Macromolecules 2018, 51, 5450-5459.

(13) Suarez-Martinez, P. C.; Batys, P.; Sammalkorpi, M.; Lutkenhaus, J. L. Time-Temperature and Time-Water Superposition Principles Applied to Poly(allylamine)/Poly(acrylic acid) Complexes. Macromolecules 2019, 52, 3066-3074.

(14) Bryant, R. G. NMR Relaxation Studies of Solute-Solvent Interactions. Annu. Rev. Phys. Chem. 1978, 29, 167-188.

(15) Waldeck, A. R.; Kuchel, P. W.; Lennon, A. J.; Chapman, B. E. NMR diffusion measurements to characterise membrane transport and solute binding. Prog. Nucl. Magn. Reson. Spectrosc. 1997, 30, 39-68.

(16) Giesecke, M.; Mériguet, G.; Hallberg, F.; Fang, Y.; Stilbs, P.; Furó, I. Ion association in aqueous and non-aqueous solutions probed by diffusion and electrophoretic NMR. Phys. Chem. Chem. Phys. 2015, 17, 3402-3408.

(17) Mo, H.; Pochapsky, T. C. Intermolecular interactions characterized by nuclear Overhauser effects. Prog. Nucl. Magn. Reson. Spectrosc. 1997, 30, 1-38.

(18) Neuhaus, D.; Williamson, M. The Nuclear Overhauser Effect In Structural And Conformational Analysis. Methods in Stereochemical Analysis, 2nd ed.; Wiley-VCH Inc., 2000.

(19) Ancian, B. Cross-relaxation and Cross-correlation Parameters in NMR: Molecular Approaches. In New Developments in NMR 12; Canet, D., Ed.; Royal Society of Chemistry, 2018; Chapter 3, pp 166-238.

(20) Hatada, K.; Kitayama, T. NMR Spectroscopy of Polymers; Springer Laboratory; Springer Berlin Heidelberg: Berlin, Heidelberg, 2004.

(21) Bagno, A.; Campulla, M.; Pirana, M.; Scorrano, G.; Stiz, S.; Padova, A.; Organica, C. Preferential solvation of organic species in binary solvent mixtures probed by intermolecular $1 \mathrm{H}$ NOESY NMR spectroscopy. Chem.-Eur. J 1999, 5, 1291-1300.

(22) Gerig, J. T. NOE Studies of Solvent-Solute Interactions. Annu. Rep. NMR Spectrosc. 2008, 64, 21-215.

(23) Li, Z.; Lenk, T. I.; Yao, L. J.; Bates, F. S.; Lodge, T. P. Maintaining Hydrophobic Drug Supersaturation in a Micelle Corona Reservoir. Macromolecules 2018, 51, 540-551.

(24) Lin, J.-H.; Chen, W.-S.; Hou, S.-S. NMR Studies on Effects of Tetraalkylammonium Bromides on Micellization of Sodium Dodecylsulfate. J. Phys. Chem. B 2013, 117, 12076-12085.

(25) Baltaze, J.-P.; Judeinstein, P. Probing ion coordination in polymer electrolytes with multinuclear NMR correlation spectroscopy. Solid State Ionics 2010, 181, 672-677. 
(26) Moreno-Villoslada, I.; González, F.; Rivera, L.; Hess, S.; Rivas, B. L.; Shibue, T.; Nishide, H. Aromatic-aromatic interaction between 2,3,5-triphenyl-2H-tetrazolium chloride and poly(sodium 4-styrenesulfonate). J. Phys. Chem. B 2007, 111, 6146-6150.

(27) Itaya, T.; Ueda, K.; Ochiai, H. Association Behavior of Hydrophobic Counterions around Poly(allylammonium) Cation. Polym. J. 1992, 24, 539-544.

(28) Bruschweiler, R.; Roux, B.; Blackledge, M.; Griesinger, C.; Karplus, M.; Ernst, R. R. Influence of rapid intramolecular motion on NMR cross-relaxation rates. A molecular dynamics study of antamanide in solution. J. Am. Chem. Soc. 1992, 114, 2289-2302.

(29) Peter, C.; Daura, X.; van Gunsteren, W. F. Calculation of NMRrelaxation parameters for flexible molecules from molecular dynamics simulations. J. Biomol. NMR 2001, 20, 297-310.

(30) Nederveen, A. J.; Bonvin, A. M. J. J. NMR Relaxation and Internal Dynamics of Ubiquitin from a $0.2 \mu \mathrm{s}$ MD Simulation. J. Chem. Theory Comput. 2005, 1, 363-374.

(31) Braun, D.; Steinhauser, O. The intermolecular NOE is strongly influenced by dynamics. Phys. Chem. Chem. Phys. 2015, 17, 8509-8517.

(32) Chalmers, G.; Glushka, J. N.; Foley, B. L.; Woods, R. J.; Prestegard, J. H. Direct NOE simulation from long MD trajectories. J. Magn. Reson. 2016, 265, 1-9.

(33) Chen, P.-c.; Hologne, M.; Walker, O.; Hennig, J. Ab Initio Prediction of NMR Spin Relaxation Parameters from Molecular Dynamics Simulations. J. Chem. Theory Comput. 2018, 14, 1009-1019.

(34) Hostnik, G.; Vlachy, V.; Bondarev, D.; Vohlídal, J.; Cerar, J. UV/ Vis Study of the Alkali Salts of Poly(thiophen-3-ylacetic acid) in Water. Acta Chim. Slov. 2012, 59, 571-581.

(35) Hostnik, G.; Bondarev, D.; Vohlídal, J.; Čebašek, S.; Žagar, E.; Vlachy, V.; Cerar, J. Transport Properties and Ion Binding in Aqueous Solutions of Alkali Metal Salts of poly(thiophen-3-ylacetic acid). J. Mol. Liq. 2014, 198, 173-180.

(36) Jeener, J.; Meier, B. H.; Bachmann, P.; Ernst, R. R. Investigation of exchange processes by two-dimensional NMR spectroscopy. J. Chem. Phys. 1979, 71, 4546-4553.

(37) States, D. J.; Haberkorn, R. A.; Ruben, D. J. A two-dimensional nuclear overhauser experiment with pure absorption phase in four quadrants. J. Magn. Reson. 1982, 48, 286-292.

(38) Bodenhausen, G.; Kogler, H.; Ernst, R. R. Selection of coherencetransfer pathways in NMR pulse experiments. J. Magn. Reson. 1984, 58, 370-388.

(39) Macura, S.; Ernst, R. R. Elucidation of cross relaxation in liquids by two-dimensional N.M.R. spectroscopy. Mol. Phys. 1980, 41, 95117.

(40) Solomon, I. Relaxation Processes in a System of Two Spins. Phys. Rev. 1955, 99, 559-565.

(41) Canet, D. Introduction: general theory of nuclear relaxation. $A d v$. Inorg. Chem. 2005, 57, 3-40.

(42) Kowalewski, J.; Mäler, L. Nuclear Spin Relaxation in Liquids: Theory, Experiments, and Applications; Series in Chemical Physics; Taylor \& Francis, 2006.

(43) Hostnik, G.; Cerar, J. On describing the equilibria in mixed solutions of polyelectrolytes and simple salts using the law of mass action. J. Mol. Liq. 2017, 228, 96-102.

(44) Manning, G. S. Limiting Laws and Counterion Condensation in Polyelectrolyte Solutions. II. Self-Diffusion of the Small Ions. J. Chem. Phys. 1969, 51, 934-938.

(45) Manning, G. S. In Polyelectrolytes, 1st ed.; Sélégny, E., Mandel, M., Strauss, U. P., Eds.; D. Reidel: Dordrecht, Netherlands, 1974; Vol. 1 , pp 9-37.

(46) Manning, G. S.; Ray, J. Counterion Condensation Revisited. J. Biomol. Struct. Dyn. 1998, 16, 461-476.

(47) Fuoss, R. M.; Katchalsky, A.; Lifson, S. The Potential of an Infinite Rod-Like Molecule and the Distribution of the Counter Ions. Proc. Natl. Acad. Sci. U.S.A. 1951, 37, 579-589.

(48) Katchalsky, A. Polyelectrolytes. Pure Appl. Chem. 1971, 26, 327374.

(49) Lifson, S.; Jackson, J. L. On Self-Diffusion of Ions in a Polyelectrolyte Solution. J. Chem. Phys. 1962, 36, 2410-2414.
(50) Gregor, H. P. In Polyelectrolytes, 1st ed.; Sélégny, E., Mandel, M., Strauss, U. P., Eds.; D. Reidel: Dordrecht, Netherlands, 1974; Vol. 1, pp $87-95$.

(51) Milne, A. W.; Jorge, M. Polarization Corrections and the Hydration Free Energy of Water. J. Chem. Theory Comput. 2019, 15, $1065-1078$.

(52) Wernersson, E.; Jungwirth, P. Effect of Water Polarizability on the Properties of Solutions of Polyvalent Ions: Simulations of Aqueous Sodium Sulfate with Different Force Fields. J. Chem. Theory Comput. 2010, 6, 3233-3240.

(53) Pluhařová, E.; Mason, P. E.; Jungwirth, P. Ion Pairing in Aqueous Lithium Salt Solutions with Monovalent and Divalent Counter-Anions. J. Phys. Chem. A 2013, 117, 11766-11773.

(54) Bhowmik, D.; Malikova, N.; Mériguet, G.; Bernard, O.; Teixeira, J.; Turq, P. Aqueous solutions of tetraalkylammonium halides: ion hydration, dynamics and ion-ion interactions in light of steric effects. Phys. Chem. Chem. Phys. 2014, 16, 13447-13457.

(55) Huš, M.; Urbič, T. Quantum Chemical Tests of Water-Water Potential for Interaction Site Water Models. Acta Chim. Slov. 2012, 59, $541-547$.

(56) Huš, M.; Urbic, T. Core-softened fluids as a model for water and the hydrophobic effect. J. Chem. Phys. 2013, 139, 114504.

(57) Huss, M.; Urbic, T. Thermodynamics and the hydrophobic effect in a core-softened model and comparison with experiments. Phys. Rev. E: Stat., Nonlinear, Soft Matter Phys. 2014, 90, 022115.

(58) Krämer, A.; Pickard, F. C.; Huang, J.; Venable, R. M.; Simmonett, A. C.; Reith, D.; Kirschner, K. N.; Pastor, R. W.; Brooks, B. R. Interactions of Water and Alkanes: Modifying Additive Force Fields to Account for Polarization Effects. J. Chem. Theory Comput. 2019, 15, $3854-3867$.

(59) Huang, J.; Lopes, P. E. M.; Roux, B.; MacKerell, A. D. Recent Advances in Polarizable Force Fields for Macromolecules: Microsecond Simulations of Proteins Using the Classical Drude Oscillator Model. J. Phys. Chem. Lett. 2014, 5, 3144-3150. 